\title{
Environmental factors influencing the distribution and prevalence of Schistosoma haematobium in school attenders of ILembe and uThungulu Health Districts, KwaZulu-Natal Province, South Africa
}

\author{
Nkosinathi Banhela ${ }^{a *}$, Myra Taylora, Siphosenkosi Gift Zulua ${ }^{(D}$, Linnea Sund Strabo ${ }^{\text {, Eyrun Floerecke Kjetland }}{ }^{\text {ab }}$ and Svein Gunnar \\ Gundersen $^{\text {cd }}$
}

\author{
${ }^{a}$ Discipline of Public Health Medicine, Nelson R Mandela School of Medicine, University of KwaZulu-Natal (UKZN), Durban, South Africa \\ ${ }^{b}$ Norwegian Centre for Imported and Tropical Diseases, Department of Infectious Diseases Ullevaal, Oslo University Hospital, Oslo, Norway \\ 'Research Department, Sorlandet Hospital HF, Kristiansand, Norway
}

${ }^{d}$ Department of Global Development and Planning, University of Agder, Kristiansand, Norway

*Corresponding author, email:nathi5sifeukzn@gmail.com

Schistosoma haematobium infection is reported to facilitate the development of urogenital diseases. Its symptoms include haematuria, dysuria and tiredness, and it may cause cognitive decline in children. The prevalence of S. haematobium infection needs to be known in endemic areas and a mass treatment programme against the disease implemented.

The aim of this study was to investigate the prevalence and intensity of S. haematobium infection in ILembe and uThungulu health districts, using the major symptom, haematuria, as an indicator. A total of 6265 urine samples, from 96 rural schools, was collected for analysis using dipsticks. The prevalence of haematuria in the ILembe health district was $37 \%(95 \% \mathrm{Cl}, 35-39 \%)$ for boys and $39 \%(95 \% \mathrm{Cl}, 37-41 \%)$ for girls. The prevalence of haematuria in the uThungulu health district was $56 \%(95 \% \mathrm{Cl}, 53-$ $59 \%)$ and $53 \%(95 \% \mathrm{Cl}, 50-56 \%)$ for girls and boys, respectively. Light-intensity infection was the most common infection level in both health districts. A negative relationship was observed between prevalence and altitude $(r=-0.262, p=0.009)$; whereas, we found a slight, though significant, positive association with mid-summer temperatures $(r=0.234, p=0.021)$. Associations between prevalence and distance of school to the nearest river were non-significant.

Keywords: altitude, haematuria, intensity, prevalence, Schistosoma haematobium, temperature, urogenital schistosomiasis

\begin{abstract}
Introduction
The neglected tropical disease of urogenital schistosomiasis is a public health challenge affecting both sexes in many developing countries. South Africa is one of the countries endemic for urogenital schistosomiasis.' An estimated 750 million people worldwide and 261 million people in sub-Saharan Africa are infected by this disease. ${ }^{2}$ Urogenital schistosomiasis is a waterborne disease caused by the parasite Schistosoma haematobium, which is carried by a specific intermediate host snail. ${ }^{3}$ People exposed to fresh water containing the infected snails are at risk of skin penetration by cercariae, which develop in the human host into adult flukes that produce eggs, causing inflammation that affects the urogenital tract. The disease has deleterious effects and symptoms, which include anaemia, tiredness and learning/work problems and target organ dysfunction., Morbidity from urogenital schistosomiasis is manifested by blood in urine (haematuria), pain when urinating (dysuria) and frequent urination, in addition to genital symptoms like discharge and dyspareunia. ${ }^{4}$ This disease may be a predisposing factor for acquisition of human immunodeficiency virus (HIV). ${ }^{16,32}$
\end{abstract}

Schistosomiasis can be diagnosed by microscopy (eggs in urine), serology, or molecular methods (such as polymerase chain reaction, $\mathrm{PCR}$ ); but, blood in urine is a common symptom of urogenital schistosomiasis; and, detection of haematuria may be an indication of S. haematobium infection, although this is controversial in females of reproductive age..$^{2,435}$ The urine reagent strip detects haematuria, and is used as a proxy diagnostic method for S. haematobium infection in endemic areas. ${ }^{7}$ The World Health Organisation (WHO) recommends mass treatment of schistosomiasis in endemic regions, with a particular focus on children, who usually have the highest prevalence and intensity of infection. ${ }^{2,8,9}$ Although studies were undertaken in this area of KwaZulu-Natal (KZN) in the 1980s and 1990s, ${ }^{10,11}$ little information is available for the last 20 years. S. haematobium is more common in areas of low socio-economic status; ${ }^{2-4}$ that is, in disadvantaged areas where water, sanitation and health facilities are inadequate. ${ }^{9}$ This poses a challenge for the rapid diagnosis, treatment and control of S. haematobium infection. The urine reagent strips (dipsticks) are easy to use, convenient and time-efficient.9,12 People in disadvantaged areas are at risk of infection because they often lack adequate safe water sources and are thus compelled to be in contact with river or dam water contaminated by $S$. haematobium..$^{2,13}$ Children playing, women doing laundry and farm workers involved in agricultural and fishery industries are at a heightened risk due to frequent contact with fresh water. $6,13,14$ Furthermore, even if the patients go to the clinic, the necessary laboratory services may not be available and patients may never return for their results due to financial, social or logistic constraints.

Urogenital schistosomiasis is a latent and chronic disease, meaning that the damage it exerts develops over time as the eggs that the adult worms produce disrupt various tissues and organs. ${ }^{4,15}$ The parasite eggs trapped in the body can induce adverse immunomodulatory effects that have been hypothesised to favour the transmission and progression of other diseases. ${ }^{16,17,25}$ Parasite eggs are small, can embolise in the bloodstream, and have the potential to breach membrane tissues that provide barrier protection against parasite, bacterial and viral entry., ${ }^{46-19}$ Research indicates that several diseases, such as cancer, HIV and 
chronic kidney disease, may be associated with S. haematobium infection. ${ }^{15,16,20,32}$ The prevalence and intensity of urogenital schistosomiasis is determined by factors such as age, geographical location, contact with contaminated water bodies, season (transmission usually occurs during the hot and humid summer) and thse distribution of the intermediate host snail. ${ }^{13,21}$

Environmental factors can serve as indicators in areas that could be at risk for S. haematobium infection. ${ }^{30}$ These environmental factors include temperature and altitude, which can influence the survival and reproduction of the intermediate host snail. ${ }^{22}$ In order for the host snail to survive, breed and complete its life cycle, these factors should occur within tolerable ranges.6,22,33 The aim of this study was to investigate the prevalence and intensity of S. haematobium in the ILembe and uThungulu health districts and association with abiotic factors, such as summer temperature, altitude above sea level and distance from the school to the nearest river.

\section{Methods}

\section{Study Setting and Design}

Neighbouring ILembe and uThungulu health districts are situated on the east coast of KZN, north of the metropolitan city of Durban. All rural high schools with more than 300 pupils were stratified by size and invited to the study, starting from the coastline and moving gradually inland. Urine samples were collected from 96 high schools (71 schools from ILembe and 25 schools from uThungulu health districts); all learners in grade eight were invited to participate in the study. Grade eight learners are usually between 13 and 16 years of age. ${ }^{6}$ All girls participating in the study were discreetly asked by a female research assistant whether they were menstruating, and the urine samples of those who were menstruating were not included in this study. In a cross-sectional design, urine samples were collected between years 2012 and 2013

\section{Ethical considerations}

Permission for the study was obtained from the Biomedical Research Ethics Committee (BREC) of the University of KwaZuluNatal (UKZN), clearance number BE165/15. Written informed consent was obtained from the parents of participating learners and learners provided assent. Treatment was offered to the ILembe and uThungulu health districts by the Department of Health in a mass-treatment campaign.

\section{Urine analyses}

Single urine samples collected in the middle of the day were tested for haematuria using urine reagent strips (Neotest 4, Occidem Biotech, UK) as an indicator of urogenital schistosomiasis. ${ }^{7}$ Haematuria, if present, was categorised as low/ light, medium/moderate, or high/heavy, corresponding to 1+, $2+$, and $3+$ readings, respectively, of reagent strips. ${ }^{6}$

\section{Temperature data}

The temperature data were obtained from the records of the South African Weather Services (SAWS). Temperature data were extrapolated from meteorological stations at Mandini [02716992], Mtunzini [03043576], Babanango [03051346] and Richards Bay Airport [03373825].

\section{Geographical Information}

The altitude and the distance of schools to the nearest river were calculated using a geographical information system (GIS) programme (ArcMap 9.2), using the geographical co-ordinates for each school provided by the Department of Education website. ${ }^{34}$
The altitude data was categorised into low (0-300 m), medium (300-800 m), high (800-1 $100 \mathrm{~m})$ and very high $(1100+\mathrm{m}){ }^{6}$

\section{Method of extracting altitude, distance values and temperature for each school}

A 20 m digital elevation model (DEM) of KZN was used to calculate the altitude of each school in the study. The elevation value represents the altitude above sea level in metres. In order to calculate the distance from each school to its closest perennial river, a spatial join was performed in ArcMap between the Rivers (1:50 000 perennial rivers dataset) and Schools shapefiles. This calculates and adds a distance field to the attribute table. This indicates the distance from the school to the closest river contact point in the GIS programme. ${ }^{36}$ The average maximum and minimum summer temperature for each weather station was calculated for the summer months (December, January and February). These values were then used to interpolate average maximum and minimum summer raster coverages for the study area, using the inverse distance weighted (IDW) spatial analyst technique in $\operatorname{ArCGIS}^{36}$

\section{Statistics}

All statistical analyses were performed using Statistical Package for Social Sciences (SPSS) 21 (Armonk, NY, USA). Descriptive analyses were undertaken for all the variables in the study. The mean prevalence of urine samples testing positive for haematuria were compared for boys and girls, using school, class per grade, health district, altitude and distance from the nearest river/s. The Kolomogorov-Smirnov test was used as a goodness-of-fit test. For data that were normally distributed, the student t-test was used and for non-normally distributed data, the Mann-U-Whitey test was used to compare averages. To establish presence and the strength of any relationship between these variables, the Spearman's correlation coefficient $(r)$ was calculated. A $p$-value of $p<0.05$ was used as the level of significance and the $95 \%$ confidence intervals were calculated.

\section{Results}

\section{Prevalence}

The total sample size was 6265 , and the prevalence of haematuria in uThungulu health district schools was 55\% (1289/2360), significantly higher than the $39 \%(1531 / 3905)$ in ILembe health district schools $(p<0.001)$. The prevalence of haematuria indicating urogenital schistosomiasis in ILembe health district was $37 \%(95 \% \mathrm{Cl}, 38-42)$ for boys and $39 \%(95 \% \mathrm{Cl}, 37-41)$ for girls $(p>0.05)$. The prevalence of haematuria in uThungulu health district was $53 \%(95 \% \mathrm{Cl}, 50-56)$ for boys and $56 \%(95 \%$ $\mathrm{Cl}, 50-56)$ for girls $(p>0.05)$ (Figure 1$)$.

In the ILembe health district, a total of 71 rural public schools were visited with totals of 1888 girls and 2017 boys tested for presence of blood in urine using reagent strips. Of those totals, 801 boys (37\%) and 730 girls (39\%) were found to have haematuria. In the uThungulu health district, a total of 25 schools were visited with 1112 girls and 1248 boys consenting to participate in the study. Of those, 626 girls (56\%) and 663 boys (53\%) were found to have haematuria.

\section{Intensity}

There were no significant differences between the sexes in low, moderate or high levels of haematuria ( $p=0.13$ ) (Figure 2). The intensities of haematuria were not significantly different between the health districts $(p>0.05)$. Although the intensity of haematuria followed the same trend for girls in uThungulu $(1+=40 \%, 2+=30 \%$, $3+=30 \%)$ and ILembe $(1+=41.3 \%, 2+=28.3 \%, 3+=30.3 \%)$, the 


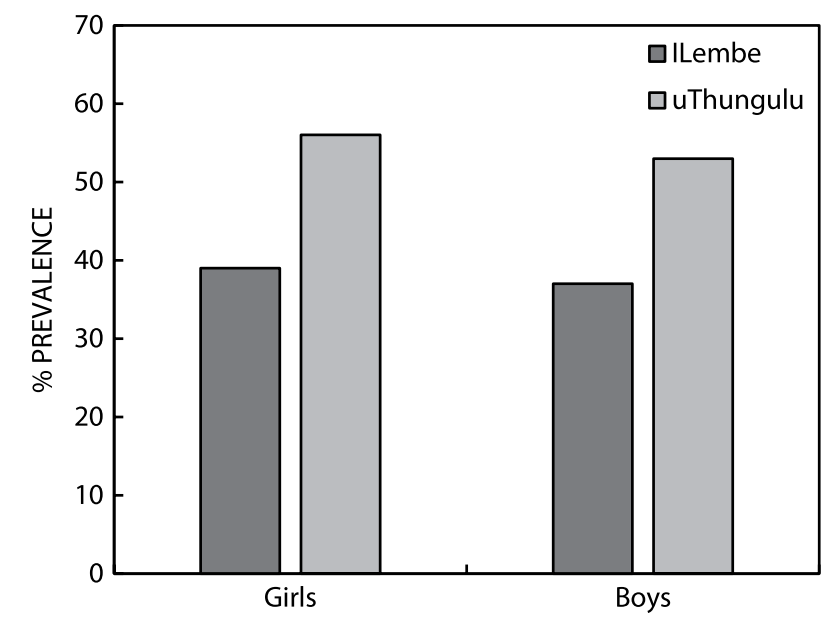

Figure 1: Prevalence of urogenital schistosomiasis by gender in grade eight learners in ILembe and uThungulu health districts, KwaZulu-Natal. $n$ (girls) = $3000 ; n$ (boys) $=3265$.

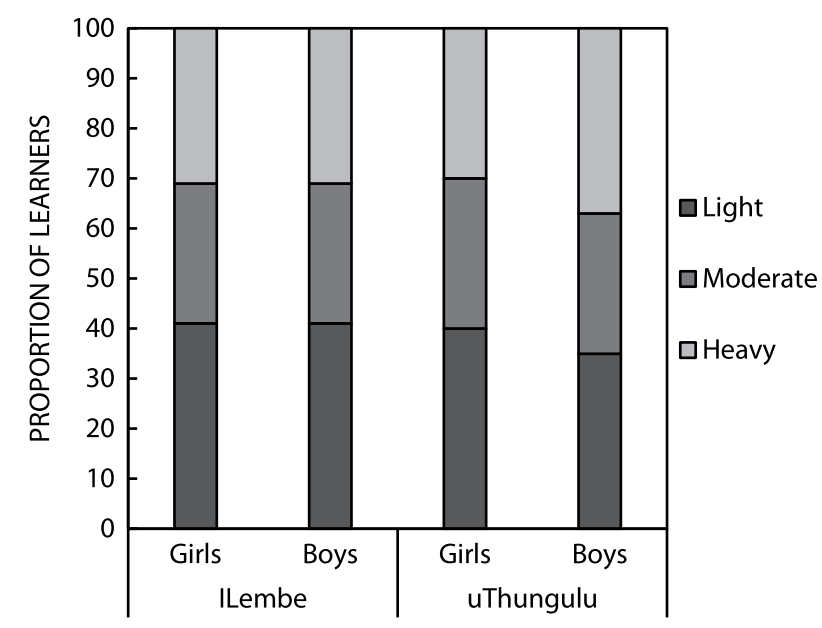

Figure 2: Mean proportions of learners per haematuria intensity category obtained from reagent strips at rural schools $(n=96)$ of ILembe (71 schools) and uThungulu ( 25 schools) Health Districts (total $n=2842$ infected learners: $n$ (girls) = $1370 ; n$ (boys) =1472).

uThungulu boys $(1+=35 \%, 2+=28 \%, 3+=36 \%)$ had a slightly higher percentage of heavy intensity haematuria compared to boys in ILembe $(1+=41 \%, 2+=28 \%, 3+=31 \%)$; although, this difference was not statistically significant $(p=0.17)$.

\section{Association of prevalence of haematuria with environmental factors}

The map (Figure 3) shows the schools in the ILembe health district (74\% of sample), where $39 \%$ of the schools are in the $0-300 \mathrm{~m}$ altitude range. In this altitude range, the majority of the schools had a haematuria prevalence greater than $20 \%$. This health district has $59 \%$ of schools in the $300-800 \mathrm{~m}$ altitude range, which showed a haematuria prevalence ranging from $11-90 \%$.

In uThungulu health district, $8 \%$ of the schools have less than 20 $\%$ prevalence and are in the $0-300 \mathrm{~m}$ altitude range, but this altitude range also includes $52 \%$ of schools that have a haematuria prevalence greater than $50 \%$. The $300-800 \mathrm{~m}$ altitude range has $36 \%$ of schools with a haematuria prevalence greater than $50 \%$. In the $800-1100 \mathrm{~m}$ altitude range in this health district, $4 \%$ of schools had a prevalence of haematuria greater than $50 \%$.
Table 1: Correlation coefficients ( $r$ ) between haematuria prevalence and associated abiotic factors (altitude above sea level, distance to nearest river contact point and the maximum and minimum average summer temperature) $(n=6265)$.

\begin{tabular}{|l|l|c|c|}
\hline Prevalence & Factor & Correlation coefficient $(r)$ & $p$-value \\
\hline Girls & $\begin{array}{l}\text { Altitude above } \\
\text { sea level }\end{array}$ & -0.215 & $0.035^{*}$ \\
\hline Boys & & -0.283 & $0.005^{*}$ \\
\hline Both & Distance to near- & -0.262 & $0.009^{*}$ \\
\hline Girls & est river & 0.010 & 0.922 \\
\hline Boys & & -0.007 & 0.947 \\
\hline Both & $\begin{array}{l}\text { Average summer } \\
\text { maximum }\left({ }^{\circ} \mathrm{C}\right)\end{array}$ & 0.008 & 0.936 \\
\hline Girls & & 0.244 & $0.017^{*}$ \\
\hline Boys & & 0.150 & 0.145 \\
\hline Both & Average summer- & 0.234 & $0.021^{*}$ \\
\hline Girls & minimum $\left({ }^{\circ} \mathrm{C}\right)$ & 0.121 & 0.242 \\
\hline Boys & & 0.146 & 0.156 \\
\hline Both & & 0.174 & 0.088 \\
\hline
\end{tabular}

${ }^{*}$ Correlation is significant at $p<0.05$.

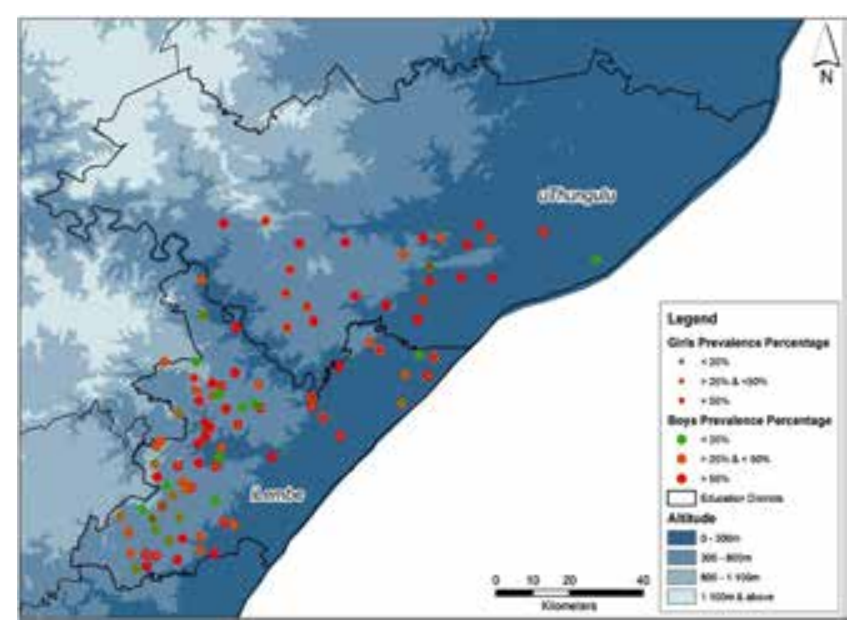

Figure 3: Map of uThungulu and ILembe health districts, categorising the prevalence $(<20 \%,>20-50 \%$ and $>50 \%)$ in boys and girls in selected schools, and the associated altitude $(\mathrm{m}) .{ }^{36}$

The altitude above sea level for the schools was significantly related to the prevalence of haematuria for both girls and boys, and the negative correlation coefficient indicates that increasing altitude was associated with a decrease in the prevalence of infection. There was a statistically significant association between the prevalence of schistosomiasis for girls and the maximum mean summer temperature $(r=0.244$, $p=0.017)$, but this was not found for boys $(r=0.150, p=0.145)$. The distance from the school to the nearest river contact point did not influence the prevalence of urogenital schistosomiasis in either sex.

There were $81 \%$ of schools in the $29.83-30.38{ }^{\circ} \mathrm{C}$ temperature range. When the prevalence of haematuria was combined for boys and girls, there was a relatively weak positive correlation $(r=0.234)$ with the average summer maximum (see Figure 4$)$, which was statistically significant $(p=0.021)$ (Table 1$)$. 


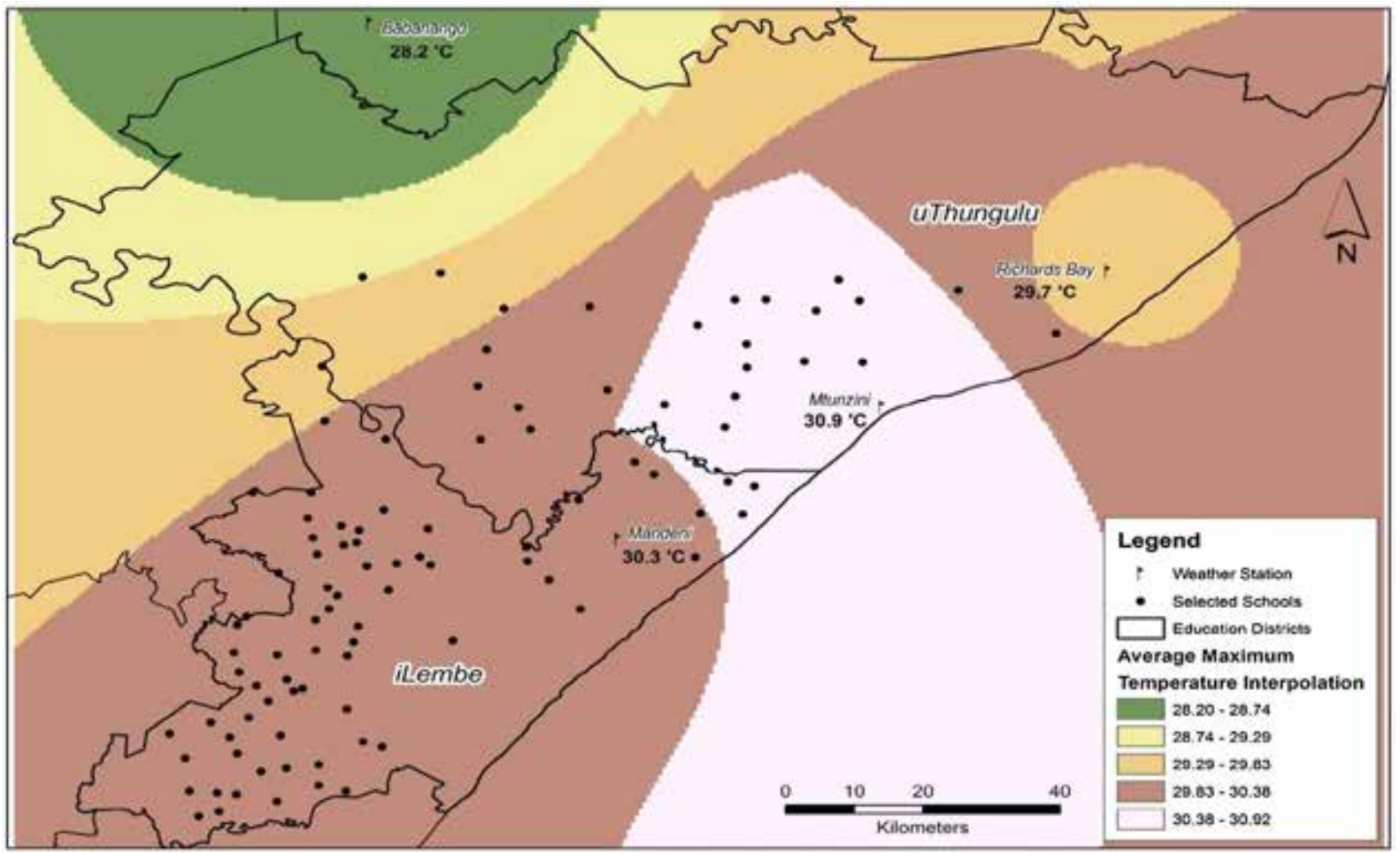

Figure 4: Average maximum summer temperature $\left({ }^{\circ} \mathrm{C}\right)$ of the areas surrounding the schools that were selected in the study..$^{36}$

\section{Discussion}

\section{Prevalence and intensity of urogenital schistosomiasis in ILembe and uThungulu health districts}

The results of this study indicate that the prevalence of infection with S. haematobium is high among school attendees. It was shown that $S$. haematobium infections are significantly higher $(p<0.001)$ in the uThungulu health district learners compared to ILembe health district learners, but sexes are equally infected in both health districts.

Learners in grade eight in the KZN province are usually between the ages of 13-16 years old, and are likely to swim or play in rivers due to the hot weather and lack of alternative recreational facilities. ${ }^{13}$ Morgas et al. noted the possibility of a higher prevalence among girls in this age group, since they are responsible for most housekeeping chores, which include washing dishes or clothes in the river and fetching water from the river for other uses. ${ }^{13}$ On the contrary, boys of this age group are at risk because they are still at an explorative stage and are eager to play or swim in river water. ${ }^{13}$

The WHO guidelines recommend that all school learners, in the prevalence category in which ILembe health district falls, receive praziquantel (PZQ) anti-schistosomiasis treatment and that this mass treatment must be done annually for at least one to two years. ${ }^{8}$ For uThungulu health district, which falls in the WHO category 1 , the recommendation would be that everyone in the community receives $\mathrm{PZQ}$ treatment irrespective of status, age or sex, and mass treatment should be provided once a year for a period of three years. ${ }^{8,24}$

Treatment of S. haematobium can have profound health improvements as destruction of adult worms by the drug can reduce the number of deposited parasite eggs. Mass treatment thus has the potential to reduce the burden of disease and its complications. ${ }^{31}$ From the association reported between female genital schistosomiasis and HIV infection, treatment of urogenital schistosomiasis may reduce the likelihood of transmission of HIV. ${ }^{16,20,32}$ The reduction of parasite egg burden in the body can reduce the damage caused by the development of sandy patches in the cervix and may reduce the development of cancer in the genitals and kidneys. . $^{13,29,32}$

In comparison, Gear et al. reported that the prevalence of urogenital schistosomiasis is $70 \%$ for schools in the ILembe health district; ${ }^{37}$ whilst, for schools in uThungulu health district, a prevalence of $26-50 \%$ was reported..$^{37}$ Over the past 30 years, the prevalence of S. haematobium has decreased by $32 \%$ in the ILembe health district, whilst it has increased by approximately $25 \%$ in the uThungulu health district. ${ }^{37}$ It is not fully understood what factors could be responsible for the change in prevalence, but changes could be due to population dynamics, geographical distributions, socioeconomic status and change in climate conditions. However, there exists an opportunity for re-infection as both health districts reported to have experienced delays in the provision of piped water and sanitation. ${ }^{23,24}$ Wolmarans et al. found the prevalence of urogenital schistosomiasis in sites in Limpopo province to be $70 \%$ in pupils younger than 14 years of age, but their sample size was smaller $(n=420)^{22,27}$ In Mpumalanga province in 2004, an epidemiological study revealed a prevalence of $35 \%$ among primary school pupils in 30 schools. ${ }^{22}$

The prevalence and distribution of urogenital schistosomiasis is influenced by environmental factors that determine the survival and replication of the intermediate host snail Bulinus africanus. ${ }^{30}$ Factors favouring or limiting B. africanus survival consequently impact S. haematobium transmission and infection. ${ }^{30}$

Most of the schools (21) in the ILembe health district have at an altitude of $300-800 \mathrm{~m}$, with a low schistosomiasis prevalence 
$(<20 \%)$, whilst schools that are located in the altitude range of 0-300 $\mathrm{m}$ tend to have an urogenital schistosomiasis infection percentage between $20 \%$ and $50 \%$ (see Figure 3). The majority of schools in uThungulu health district $(0-800 \mathrm{~m})$ showed infection rates greater than $20 \%$ and in some schools, greater than $50 \%$.

The intensity of haematuria (light, moderate or heavy) was not affected by gender differences, but intensity of infection varied by health district, with schistosomiasis infection higher in schools located in the uThungulu health district compared to schools in the ILembe health district $(p<0.005)$. In the uThungulu health district, boys had a slightly higher tendency to show heavy infection, whilst girls in this health district were predominantly in the light infection category (Figure 2). A 1+ reading on a reagent strip indicates a light intensity infection, and this is equivalent to a release of 5-10 erythrocytes per $\mu \mathrm{l}$ of urine. ${ }^{6}$ This means that $41 \%$ (see Figure 2) of boys and girls in ILembe Health District are losing 5-10 erythrocytes per $\mu$ l of urine, which can be a significant number of lost red blood cells due to haematuria. In the uThungulu health district, $36 \%$ of boys lose red blood cells in urine (around 250 erythrocytes per $\mu$ l of urine) through their heavy intensity of infection. Thirty percent of girls had a heavy intensity haematuria in uThungulu health district. Loss of blood in urine can have serious health implications, such as anaemia and lethargy. ${ }^{3,4}$

Genital lesions and membrane damage caused by trapped parasite eggs can be minimised if treatment is offered timeously; therefore, treatment of school pupils vulnerable to $S$. haematobium infection is required. Early treatment of urogenital schistosomiasis may reduce the transmission and potentiation of transmission of HIV. ${ }^{20,32}$ Treatment is required equally for both sexes as both boys and girls appear to suffer from similar rates of infection. The WHO has recommended the joint delivery of drugs for both schistosomiasis and soil-transmitted helminth infections, after it was observed that these two infections threaten similar communities and are most likely to be found where there is no piped water and improved sanitation..$^{25}$ With school-going learners at the highest risk for infection, regular prophylactic treatment is advised. This could benefit schoolgoing children infected with $S$. haematobium by lowering the incidence of anaemia and the associated lethargy, and improving their ability to focus in school; hence, improve their school attendance. ${ }^{25}$ Since this is a neglected disease, local health facilities in rural areas might lack equipment for diagnosis and sometimes PZQ might not be available at primary healthcare clinics. For these reasons mass treatment with PZQ at schools endemic for S. haematobium infection is encouraged. ${ }^{2,5,25}$ The use of urine reagent strips can assist the diagnosis process, if needed. ${ }^{9}$

In this study, the prevalence and intensity of urogenital schistosomiasis was measured using urine reagent strips. Urine reagent strips designed to detect active $S$. haematobium infection are estimated to have a sensitivity and specificity of $82 \%$ and $97 \%$, respectively. ${ }^{12}$ This suggests that the results presented here can be trusted, as there is no significant difference between infection levels detected with microscopy or urine reagent strip. ${ }^{6,27}$ However, false positives can arise from bacterial or viral infection(s) that cause haematuria, and if menstruating girls are tested. ${ }^{7,14}$ The prevalence presented in this research represents data from only the school-going population and may not be a good representation of whole populations of the health districts studied herein.

\section{Relationship between prevalence and environmental factors}

The prevalence of urogenital schistosomiasis showed a significant negative relationship with altitude above sea level in boys and girls ( $p=0.035$ and 0.005 , respectively) (see Table 1). The Spearman's rank correlation showed a weak negative correlation coefficient for the relationship between altitude and prevalence of infection for girls and boys $(r=-0.215$ and -0.283 , respectively). This finding is congruent with what other researchers have found, in that locations of low altitude (around $250 \mathrm{~m}$ ) are conducive for the survival and reproduction of the intermediate snail host; and, hence, such areas have a high prevalence of $S$. haematobium..$^{28}$ However, the negative relationship observed between altitude and prevalence of urogenital schistosomiasis is not absolute, as there are schools at the $0-300 \mathrm{~m}$ altitude with low prevalence and there are also schools at 300-800 $\mathrm{m}$ with high prevalence.

The relationship between prevalence of urogenital schistosomiasis and distance to the nearest river was not significant for either girls or boys ( $p=0.922$ and 0.947, respectively). Although, in this study there was no statistically significant association between school location and the distance to the nearest river with urogenital schistosomiasis prevalence, one study reported that learners at schools that were close to rivers and/or dams had a higher prevalence of $S$. haematobium infection than those at schools further away. ${ }^{29}$ In the present area, the distances between the homes and the rivers might be of more importance.

Associations between average summer maximum $\left({ }^{\circ} \mathrm{C}\right)$ temperature and prevalence of urogenital schistosomiasis showed a weak positive relationship for both girls and boys ( $r=0.244$ and 0.150 , respectively). This relationship was significant for girls $(p=0.017)$ but it was non-significant for boys $(p=0.145)$. When the prevalence was combined for girls and boys, a positive correlation $(r=0.234)$ was observed that was significant ( $p=0.021$ ) (Table 1). Annual temperature ranges in ILembe and uThungulu health districts are adequate for the survival and breeding of the host snail, since the prevalence of haematuria due to urogenital schistosomiasis was moderately high (37-56\%).

\section{Conclusion}

Urine reagent strips have proved useful in the identification and quantification of haematuria as a proxy for urogenital schistosomiasis infection. Urogenital schistosomiasis infections are moderately high among the school going population of ILembe and uThungulu health districts. Early treatment of urogenital schistosomiasis can prevent the development of genital lesions and kidney damage that is due to the calcification of parasite eggs and formation of fibrous tissue in genitals and the kidneys. The environmental factors explored in this study (temperature and altitude) can serve as useful indicators in the identification of areas at risk for urogenital schistosomiasis.

Acknowledgments - We would like to thank Prof Christopher Appleton for scientific advice on bilharzias he offered. Michella Du Sart of EduAction for GIS advice and maps. The Department of Statistics, Uppsala University, for statistical advice.

The authors are also indebted to the South African Weather Services (SAWS) and the National Institute of Communicable Diseases (NICD). 
Funding - Funding for the study was received from the University of KwaZulu-Natal, College of Health Sciences.

Supplementary funding was received from the Erasmus Mundus (INSPIRE) scholarship.

\section{ORCID}

Siphosenkosi Gift Zulu (iD) http://orcid.org/0000-0002-4996-8827

\section{References}

1. Johnson CC, Appleton CC. Urban schistosomiasis transmission in Pietermaritzburg, South African. South Afr J Epidemiol Infect. 2005;20(3):103-7.

2. World Health Organization. Technical report series. Prevention and control of schistosomiasis in endemicareas. Geneva: World Health Organization; 2012. 87, 81-9.

3. Brooker S. Spatial epidemiology of human schistosomiasis in Africa: risk models, transmission, dynamics and control. Trans Royal Soc Trop Med Hyg. 2007;101(1):1-8. doi:10.1016/j.trstmh.2006.08.004.

4. Barsoum RS, Esmat G, El-Baz T. Human schistosomiasis: clinical perspective. J Adv Res. 2013;4(5):433-44. Available 2013 Aug 8 from: https://doi.org/10.1016/j.jare.2013.01.005

5. Amazigo UV, Leak SGA, Zoure HGM, et al. Community-driven interventions can revolutionise control of neglected tropical diseases. J Negl Trop Dis. 2012;26(6):231-8.

6. Appleton CC, Kvalsvig JD. A school-based helminth control program successfully implemented in Kwa-Zulu-Natal. South Afr J Epidemiol Infec. 2006;21(2):55-67.

7. Pugia MJ. Technology behind diagnostic reagent strips. Lab Med. CE Update Instrumentation ii. 2000;31(2):46515-0070.

8. World Health Organization. Technical report series. Prevention and control of schistosomiasis and soil transmitted helminths. Geneva: World Health Organization; 1998. 813-911.

9. Taylor M, Jinabhai CC, Naidoo K, et al. The epidemiology of schistosomiasis among Zulu children in a rural district in South Africa: determining appropriate community-based diagnostic tools. South Afr J Epidemiol Infect. 2004;19(3, 4):90-5.

10. Pitchford RJ. Temperature and schistosome distribution in South Africa. South Afr J Sci. 1981;77:252-61.

11. Schutte CHJ, van Deventer JGM, Lamprecht T. A cross-sectional study of the prevalence and intensity of infection with Schistosoma haematobium in students of northern KwaZulu-Natal. Am JTrop Med Hyg. 1981;30:364-72. https://doi.org/10.4269/ajtmh.1981.30.364

12. King $\mathrm{CH}$, Bertsch D. Meta-analysis of urine heme dipstick diagnosis of Schistosoma haematobium infections including low prevalence and previously-treated populations. PLoS Neglect Trop Dis. 2013;7(9):e2431. doi:10.1371/journal.pntd.0002431.

13. Morgas DE, Kvalsvig JD, Gunderson SG, et al. Schistosomiasis and water-related practices in schoolgirls in rural KwaZulu-Natal, South Africa. South Afr J Epidemiol Infect. 2010;25(4):31-3.

14. Freeman MC, Clasen T, Brooker SJ, et al. The impact of school-based hygiene, water quality and sanitation, intervention on soil-transmitted helminth reinfection: a cluster randomized trial. Am J Trop Med Hyg. 2013;89(5):875-83. https://doi.org/10.4269/ajtmh.13-0237

15. Kayange NM, Smart LR, Tallman JE, et al. Kidney disease among children in sub-Saharan Africa. A systematic review. Paediat Res. 2015;77(2):85-9. doi:10.1038/pr2015.189.

16. Mbabazi PS, Andan O, Fitzgerald DW, et al. Examining the relationship between urogenital schistosomiasis and HIV infection. PLoS Neglect Trop Dis. 2011;5(12):e1396. doi:10.1371/journal.pntd.0001396.

17. Jourdan PM, Roald B, Poggensee G, et al. Increased vascularity in cervicovaginal mucosa with Schistosoma haematobium infection. PLos Neglect Trop Dis. 2011;5(6):1-6.

18. Kildemoes AO, Kjetland EF, Zulu SG, et al. Schistosoma haematobium infection and asymptomatic bacteriuria in young South African females. Acta Tropica 2015;144:14-23. doi:10.10.16/j. actatropica.2015.01.008.
19. Senghor B, Diallo A, Doucoure SS, et al. Prevalence and intensity of urinary schistosomiasis among school children in the District of Niakhar, Region of Fatick, Senegal. Parasit Vectors 2014;7:5. doi:10.1186/1756-3305-7-5.

20. Hotez JP, Molyneux DH, Fenwick A, et al. Incorporating a rapid-impact package for neglected tropical diseases with programs for HIV/AIDS, tuberculosis, and malaria. A comprehensive pro-poor health policy and strategy for the developing world. PloS Med. 2006;3(5):0576-84.

21. Saathoff E, Olsen A, Magnessen P, et al. Patterns of Schistosoma haematobium infection, impact of PZQ treatment and re-infection after treatment in a cohort of school children from rural KwaZuluNatal. BMC Infect Dis. 2004;4:1. doi:10.118611471-2334-4-40.

22. De Kock KN, Wolmarans CT. Distribution and habitats of the Bulinus africanus species group, snail intermediate hosts of Schistosoma haematobium and S. mattheei in South Africa. Water SA 2004;31(1):117-27.

23. ILembe District Municipality. Integrated development plan. Annual Review. (2011/2012).

24. UThungulu District Municipality. Integrated development plan. (2012/2013).

25. Randrianasolo BS, Jourdan PM, Ravoniarimbinina $\mathrm{P}$, et al. Gynecological manifestations, histopathological findings and schistosoma-specific PCR results among women with Schistosoma haematobium infection: a cross-sectional study in Madagascar. J Infect Dis. 2015;212(2):275-84. doi: 10.1093/infdis/jiv035.

26. World Health Organization (WHO). Health of school children. Treatment of intestinal helminths and schistosomiasis. WHO/schisto/95.112; 1995.

27. Meents EF, Boyles TH. Schistosoma haematobium prevalence in school children in the rural Eastern Cape Province, South Africa. South Afr J Epidemiol Infect. 2010;25(4):28-9.

28. Liao CW, Sukati $H$, Nara $T$, et al. Prevalence of Schistosoma haematobium infection among schoolchildren in remote areas devoid of sanitation in north western Swaziland, Southern Africa. Japan J Infect Dis. 2011;64:322-6.

29. Oniya MO, Ishola MA, Jayeoba OD. Schistosomiasis in Ipogun: update assessment on endemicity and efficacy of praziquantel in chemotherapy. Int J Trop Dis Health 2013;3(1):37-44.

30. Appleton CC. Review on abiotic factors influencing the distribution and the life cycles of bilharziasis host snail. Malacol Rev. 1978;11:1-25.

31. World Health Organization/Pan American Health Organization (WHO/PAHO). Schistosomiasis regional meeting: defining the road map toward verification of elimination of schistosomiasis in Latin America and the Caribbean by 2020 [internet]. 2014. Available 2016 Feb 11 from: www.paho.org/hq/index.php?option=com topics\&view=rdmore\&cid=6157\&ltemid=40770\&lang=en

32. Kjetland EF, Leutscher PDC, Ndhlovu PD. A review of female genital schistosomiasis. Trends Parasitol 2012;28(2):1-4.

33. Joubert PH, Pretorius SJ, de Kock KN, et al. Survival of Bulinus africanus (Krauss), Bulinus globosus (Morelet) and Biomphalaria pfeifferi (Krauss) at constant high temperatures. South Af J Zool. 1986;21(1):85-8. doi:10.10801/02541858.1447963.

34. Department of Education KwaZulu-Natal school list. 2012. Available 2015 Sep 03 from: www.kzneducation.gov.za/portals/EMIS/KZN\%20 List\%202012.xls

35. Gundersen SG, Kjetland EF, Poggensee G, et al. Urine reagent strips for diagnosis of Schistosoma haematobium in women of fertile age. Acta Tropica 1996;62:281-7. https://doi.org/10.1016/S0001706X(96)00029-0

36. Edu-Action. GIS and Education Consultants. Available 2015 Mar 3 from: www.eduaction.co.za

37. Gear JHS, Pitchford RJ, van Eeden JA. Atlas of Bilharzia in Southern Africa. South African Institute for Medical Research, South African Medical Research Council and Department of Health. Johannesburg: SAIMR;1980. 